\title{
Symmetries, Topological Phases and Bound States in the One-Dimensional Quantum Walk
}

\author{
J. K. Asbóth \\ Institute for Solid State Physics and Optics, Wigner Research Centre, \\ Hungarian Academy of Sciences, H-1525 Budapest P.O. Box 49, Hungary
}

(Dated: Autumn 2012)

\begin{abstract}
Discrete-time quantum walks have been shown to simulate all known topological phases in one and two dimensions. Being periodically driven quantum systems, their topological description, however, is more complex than that of closed Hamiltonian systems. We map out the topological phases of the particle-hole symmetric one-dimensional discrete-time quantum walk. We find that there is no chiral symmetry in this system: its topology arises from the particle-hole symmetry alone. We calculate the $\mathbb{Z}_{2} \times \mathbb{Z}_{2}$ topological invariant in a simple way that is consistent with a general definition for 1-dimensional periodically driven quantum systems. These results allow for a transparent interpretation of the edge states on a finite lattice via the the bulk-boundary correspondance. We find that the bulk Floquet operator does not contain all the information needed for the topological invariant. As an illustration to this statement, we show that in the split-step quantum walk, the edges between two bulks with the same Floquet operator can host topologically protected edge states.
\end{abstract}

\section{INTRODUCTION}

The quantum mechanical generalization of the random walk has, since its first definition ${ }^{1}$ received quite some interest. Its hallmark property is that as opposed to the classical walk, the standard deviation of the position of the walker increases linearly with time. This $\sqrt{t}$ speedup over the classical diffusive scaling lies behind the advantage of the Grover search. This is all the more interesting, as a variant of the quantum walk can realize a general purpose quantum computer ${ }^{213}$. Quantum walks have also attracted attention as a convenient platform to study the effects of decoherence ${ }^{4}$. The surge of interest in quantum walks has resulted in their experimental realization in varied physical systems, such as trapped ions 5 , cold atoms in optical lattices ${ }^{7}$, and on photons on an optical table 8 .

A discrete time, coined quantum walk can be viewed as a stroboscopic simulation of time evolution by an effective Hamiltonian. The topological features of lattice Hamiltonians has in the last decade been the focus of intense interest in solid state physics. The so-called bulk-boundary correspondence, showing how differences between bulk topologies give rise to low-energy states residing at the "edges", the boundaries between these bulks, is at the heart of the general theory of topological insulators10111. Recently, Kitagawa et al. have shown how, by varying the parameters of the discrete-time quantum walk, one can realize all known kinds of topological phases in 1 and 2 dimensions $\mathbf{1 2}^{1213}$. The striking physical consequence is that in an inhomogeneous system, a walker started at a boundary between domains with different topology can be localized (1D case), or propagate unidirectionally (2D case ${ }^{\sqrt{12}}$. This "trapping effect" for a 1D quantum walk has already been seen in an experiment performed with photons 14 . The appearance of these "trapping states" in a disordered quantum walk can lead to sub-diffusive spreading of the wavefunction $\sqrt[815]{ }$, a phenomenon familiar from disordered superconducting wires $\frac{16}{}$.

In the lab, a discrete-time quantum walk is realized by periodically modulating the parameters of the experimental setup. Compared to a closed, time-independent system, a quantum walk thus can have a broader range of ways in which topology can enter its description. One example is the winding of quasienergy ${ }^{17}$, which can lead to novel kinds of edge states. However, even for the quantum walks where the winding of the quasienergy is 0 , it is a relatively unexplored question to what extent their topological properties go beyond that of the underlying effective Hamiltonian.

The bulk-boundary correspondence predicts "edges states" where the edges are defined by assigning a position dependence to the parameters of a system. In many practical situtations, however, an edge represents the physical boundary of the system. For Hamiltonian systems, the simplest approach, called "open boundary conditions", is to set the hopping rates to zero at the edge. For quantum walks, boundaries are realized by using reflective coins, or - the analogue of open boundary conditions - cutting the links. Topologically protected states at such boundaries have been predicted ${ }^{15 \mid 18}$, and analyzed using an adiabatic argument $\frac{13}{}$. However, their relation to the bulk-boundary correspondence is so far not understood.

In this paper, we revisit the question of the topological phases of the 1-dimensional discrete-time quantum walk. In Section II] we define the quantum walk that we are going to study, along with the introduction of the associated effective Hamiltonian. In Section III we analyze the symmetries of the system. Our choice of coin operator, which is widely used ${ }^{12|13| 15}$ ensures Particle-Hole Symmetry of the effective Hamiltonian. However, contrary

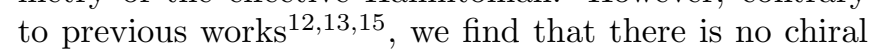
symmetry for this walk. We also discuss the sublattice symmetry of the time evolution operator: this turns out to cause energy eigenstate to come in pairs, but other- 
wise have no significant consequences for the topology. In Section [V] we explore the topological phases of the quantum walk. At variance with Kitagawa et al $\frac{12}{12}$, we find two different topological phases for the simple 1D quantum walk. A spatial boundary between domains with different topology hosts a pair of topologically protected bound states. We show that a naive way to determine the relative values of these invariants is in line with the definition of the topological invariant for periodically driven quantum systems due to Jiang et al!19?

In Section $\mathrm{V}$ we consider the quantum walk on a finite line. Termination of the lattice by a completely reflective coin operator and "open boundary conditions" by cutting the links have already been considered, but we rederive the results using the bulk-boundary correspondence for completeness. Cutting the links at the boundary leads us to a generalization of the discrete time quantum walk which is equivalent to the split-step walk ${ }^{12}$. We find that the split-step walk has a $\mathbb{Z}_{2} \times \mathbb{Z}_{2}$ topological invariant, which is unique to periodically driven quantum systems. We map out the parameter space of the split-step walk. This allows us to predict that a generic 1D particle-holesymmetric discrete time quantum walk has a single topologically protected edge state at each "open boundary", with energy $E=0$ or $E=\pi$, depending on the topology of the bulk and on how the link at the boundary is cut. This is in contrast to boundaries defined by reflective coins, where either a pair of bound states with energies $E=0$ and $E=\pi$ are present, or no bound states at all. Finally, we provide a striking example of the way in which periodically driven systems have topological features not present in their effective lattice Hamiltonians: A boundary between two quantum walks with the same bulk timestep operator supporting a pair of edge states with energies $E=0$ and $E= \pm \pi$.

\section{DISCRETE TIME QUANTUM WALK}

The quantum walk we consider in this paper is a standard extension of the common discrete-time quantum walk. We consider a particle with a discrete position degree of freedom, $x=0, \ldots, N$, and two internal (coin) states, labeled $\uparrow$ and $\downarrow$. Thus, the quantum state of the particle can be represented by a complex $2 N$-component vector:

$$
|\Psi\rangle=\sum_{x=1}^{N}\left(\Psi_{x, \uparrow}|x\rangle \otimes|\uparrow\rangle+\Psi_{x, \downarrow}|x\rangle \otimes|\downarrow\rangle\right) .
$$

The dynamics of the quantum walk is given by a unitary timestep (Floquet) operator, consisting of a rotation of the spin followed by a spin-dependent shift of the particle,

$$
|\Psi(t+1)\rangle=U|\Psi(t)\rangle=S R|\Psi(t)\rangle .
$$

This is illustrated in Fig. 1. Conveniently, we choose the unit of time to be the period of the time evolution, the unit of position the period of the lattice, and set $\hbar=1$.

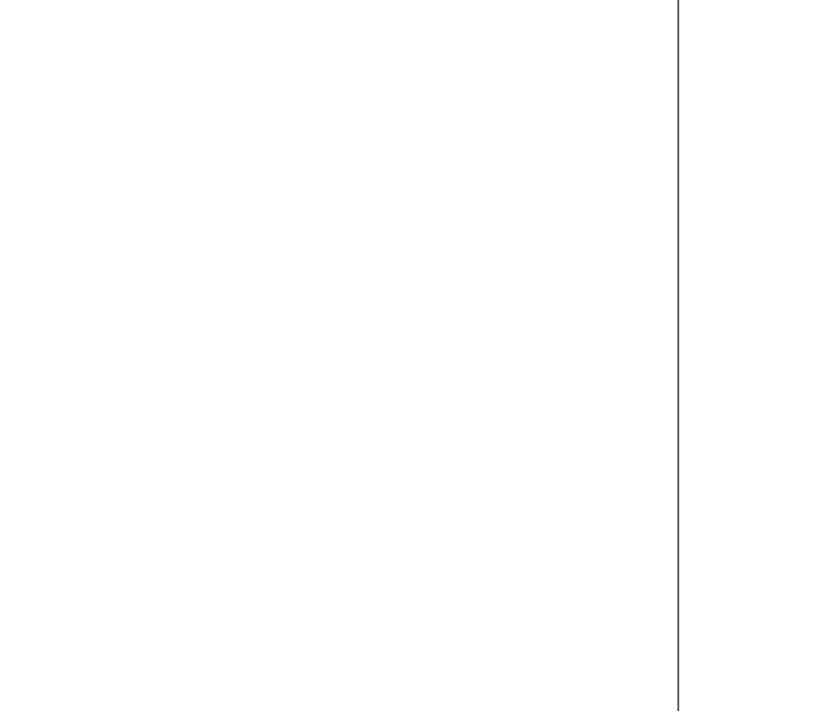

FIG. 1: The discrete-time quantum walk. A spin-1/2 particle starting from a site of a discrete lattice undergoes alternating spin rotations $R$ and spin- $z$ dependent unitary shifts $S$. The first few timesteps are shown representing the effect of interference.

The operator $S$ translates the particle by one lattice site to the left (right), if its spin is pointing down(up),

$$
S=\sum_{x=1}^{N}(|x-1\rangle\langle x|\otimes| \downarrow\rangle\langle\downarrow|+| x+1\rangle\langle x|\otimes| \uparrow\rangle\langle\uparrow|) .
$$

Periodic boundary conditions are taken, i.e., $N+1=1$. For a translation independent bulk, we use the Fourier transform, $|k\rangle=\frac{1}{\sqrt{N}} \sum_{x} e^{-i k x}|x\rangle$, and can write the particle shift operator as

$$
S=\sum_{k}\left\{e^{-i k}|\uparrow\rangle\left\langle\uparrow\left|+e^{i k}\right| \downarrow\right\rangle\langle\downarrow|\right\} \otimes|k\rangle\langle k|=e^{-i k \sigma_{z}} .
$$

Here and in the following the operators $\sigma_{x, y, z}$ denote the Pauli matrices acting on the internal "pseudospin" degree of freedom, with basis states $|\uparrow\rangle,|\downarrow\rangle$.

The coin operator $R$ is a unitary rotation in the internal space of the particle (corresponding to the "coin flip" in the classical walk.) It is diagonal in $x$,

$$
R=\sum_{x}|x\rangle\langle x| \otimes R(x) .
$$

We require $\left[R(x), R\left(x^{\prime}\right)\right]=0$ and $\sigma_{z} R(x) \sigma_{z}=R(x)^{-1}$ for every $x, x^{\prime}$, in order to ensure particle-hole symmetry (see details later). In that case, without any loss of generality, we can take $R(x)$ to be a unitary rotation of the spin around the $y$ axis by a position-dependent angle $\theta$,

$$
R(x)=R(\theta(x))=\exp \left(-i \theta(x) \sigma_{y}\right) .
$$




\section{A. Effective Hamiltonian}

To realize the quantum walk, we need an experimental setup with time-dependent external fields. Denoting the explicitly time-dependent Hamiltonian by $H(t)$, we have

$$
U=\mathbb{T} e^{-i \int^{1} H(t) d t},
$$

where $\mathbb{T}$ is the time-ordering operator. Taking the logarithm of $U$, we can associate a time-independent effective Hamiltonian $H_{\text {eff }}$ to this unitary operator (cf. Floquet theory), defined as

$$
U=e^{-i H_{\text {eff }}}
$$

In the translation invariant bulk, the time evolution operator is diagonal in momentum space, $U=\sum_{k} U(k) \otimes$ $|k\rangle\langle k|$, with

$$
U(k)=e^{-i k \sigma_{z}} e^{-i \theta \sigma_{y}}=e^{-i H_{\text {eff }}(k)} .
$$

In the bulk, the quantum walk realized by $H(t)$ stroboscopically simulates the time evolution via $H_{\text {eff }}$. The eigenvalues of the effective Hamiltonian $H_{\text {eff }}$ are the quasienergies, which can be restricted to an energy Brillouin zone $-\pi, \ldots, \pi$, in the same way as the quasimomenta are restricted to the first Brillouin zone. Since $U$ is a product of $\mathrm{SU}(2)$ operators, its determinant is 1 , thus $H_{\text {eff }}$ has to be traceless, and the spectrum has to be symmetric around $E=0$. Note that this is a property of the spectrum and not of $H_{\text {eff }}(k)$, and in itself implies neither particle-hole symmetry (ensured by our choice of $R$ ) nor chiral symmetry (absent in this system: see Section III C) of the effective Hamiltonian $H_{\text {eff. However, it does }}$ mean that there can be no winding in quasienergy 17 .

\section{SYMMETRIES AND GAPS}

To understand what topological phases and topologically protected edge states the quantum walk might have, we need to examine the symmetries and the related protected gaps of the effective Hamiltonian.

\section{A. Particle-Hole Symmetry}

The unitary timestep operator (cf Eqs. (2), (3), (6) ) in position and $\sigma_{z}$-basis has only real elements:

$$
U^{*}=U,
$$

where here and in the following $*$ denotes complex conjugation in the $x$ and $\sigma_{z}$-basis. By the definition of the effective Hamiltonian, this implies

$$
H_{\mathrm{eff}}^{*}=-H_{\mathrm{eff}} ; \quad \Longrightarrow H_{\mathrm{eff}}^{*}(-k)=-H_{\mathrm{eff}}(k)
$$

For stationary states $|\Psi\rangle$ of the walk this translates to

$$
H_{\mathrm{eff}}|\Psi\rangle^{*}=-E|\Psi\rangle^{*}
$$

Thus we have Particle-Hole Symmetry (PHS), with $P^{2}=1$. It is represented by complex conjugation: $E \leftrightarrow-E ;|\Psi\rangle \leftrightarrow|\Psi\rangle * 12$.

Eigenstates of the quantum walk with energy 0 or $\pi$ can be their own particle-hole symmetric partners - this happens if their wavefunctions are real. If there is a bulk gap around these states (if these are midgap states), their energies can be protected against particle-hole symmetric perturbations.

\section{B. Sublattice Symmetry}

The lattice on which the walk takes place is bipartite: we can assign each lattice site $j$ to one of the sublattices $\alpha$ and $\beta$, with every link connecting sites from different sublattices. Moreover, the lattice of the unitary timestep operator itself is bipartite:

$$
U=\sum_{\langle j l\rangle} U_{j l}|j\rangle\left\langle l\left|+U_{l j}\right| l\right\rangle\langle j|: j \in \alpha ; \quad l \in \beta,
$$

where the $U_{j l}=\langle j|U| l\rangle$ are operators in spin space. This leads to a symmetry of the effective Hamiltonian ${ }^{15}$, that is sometimes called "chiral symmetry" 20 . Since this symmetry arises from the bipartition of the timestep operator, we are going to call it "sublattice symmetry".

Defining the sublattice operator $\tau_{z}$, we can express sublattice symmetry in a concise way:

$$
\begin{aligned}
\tau_{z} & \equiv \sum_{j \in \alpha}|j\rangle\left\langle j\left|-\sum_{l \in \beta}\right| l\right\rangle\langle l| ; \\
\tau_{z} U \tau_{z} & =-U .
\end{aligned}
$$

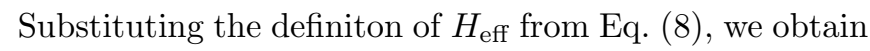

$$
\tau_{z} H_{\mathrm{eff}} \tau_{z}=H_{\mathrm{eff}}+\pi .
$$

For energy eigenstates $|\Psi\rangle$, this means

$$
H_{\mathrm{eff}} \tau_{z}|\Psi\rangle=(E+\pi) \tau_{z}|\Psi\rangle
$$

Note that $\tau_{z}$ is a local operator: we can extend the unit cell in such a way that the matrix of $\tau_{z}$ is translation invariant, and does not link different unit cells. Moreover, $\tau_{z}$ is independent of all of the angles $\theta(x)$, and so defines a unitary symmetry for the whole set of Hamiltonians $\left\{H_{\text {eff }}(\theta(x))\right\}$.

Sublattice symmetry (SLS) does not change the number of independent, symmetry protected gaps. On the one hand, SLS implies that the bulk has a gap around $E=\pi$ if and only if it has a gap around $E=0$ : This decreases the number of independent, symmetry protected gaps from 2 to 1 . On the other hand, however, there is a new kind of protected gap. For a state with with energy $\pi / 2$, its SLS partner can coincide with its PHS partner. This happens, e.g., if the wavefunction is real on even and imaginary on odd sites. Assuming there is a bulk gap around energy $\pi / 2$ (and therefore around $E=-\pi / 2$ as well), the energies of this pair of states are protected by SLS and PHS. 


\section{No chiral symmetry}

Importantly, it is the lattice of the timestep operator $U$, and not of the effective Hamiltonian $H_{\text {eff }}$, that is bipartite. If the Hamiltonian was bipartite, that would give us chiral symmetry, with a unitary operator $W=\tau_{z}$, as defined in Eq. (13), and

$$
W H_{\mathrm{eff}} W^{\dagger}=-H_{\mathrm{eff}} .
$$

Here, we find no local unitary operator $W$ representing such a symmetry.

Kitagawa et al 12 identify a "chiral symmetry" for the system, with $W=\cos \theta \sigma_{x}+\sin \theta \sigma_{z}$. However, since this operator depends explicitly on $\theta$, we do not think it should be considered a "symmetry". Whenever symmetry properties of a system are investigated, it is not only one specific Hamiltonian, but an ensemble of Hamiltonians that should be considered. The operator representing the symmetry has to be the same for all elements of the ensemble. The ensemble we consider here, are the quantum walks with varying rotation angles $\theta$. This follows from the fact that $\theta$ is the only tunable parameter of the walk that we can use, e.g., to create an inhomogeneous system with different domains. Since the "chiral symmetry operator" $W$ depends explicitly on $\theta$, it does not represent a symmetry of the system. (In an inhomogeneous system, $\theta$ is a spatially varying parameter, and so $W$ is not even properly defined.)

Since $U(k)$ has determinant $1, H_{\text {eff }}(\theta, k)$ is traceless, and therefore its spectrum is symmetric for any $\theta$. This could hint at chiral symmetry: a unitary operator $W$ that transforms the positive energy eigenstate $|+, \theta, k\rangle$ of $H_{\text {eff }}(\theta, k)$ into its negative energy eigenstate, $|-, \theta, k\rangle$, and vice versa. However, for different values of $\theta$, as $k$ is swept through $[-\pi, \pi]$, the eigenstate $|+, \theta, k\rangle$ takes on every value on different great circles on the Bloch sphere ${ }^{12}$. Unitary transformations are rotations on the Bloch sphere, and there is no rotation that takes every point to its antipodal pair on two different great circles. Therefore there is no chiral symmetry for the effective Hamiltonian of the discrete time quantum walk. Since we have particle-hole symmetry, the absence of chiral symmetry also precludes the existence of time reversal symmetry of the effective Hamiltonian.

\section{TOPOLOGICAL PHASES OF THE QUANTUM WALK}

To understand the topological phases of the quantum walk, we treat the translation independent (bulk) case, i.e., $\theta(x)=\theta$ independent of $x$. The dispersion relation of the effective Hamiltonian follows from eq. (9) in a straightforward way 12 ,

$$
\cos E(k)=\cos (k) \cos (\theta) .
$$

The resulting dispersion relations for generic values of $\theta$, and for the special values $\theta=0$, and $\theta=\pi$ are plotted

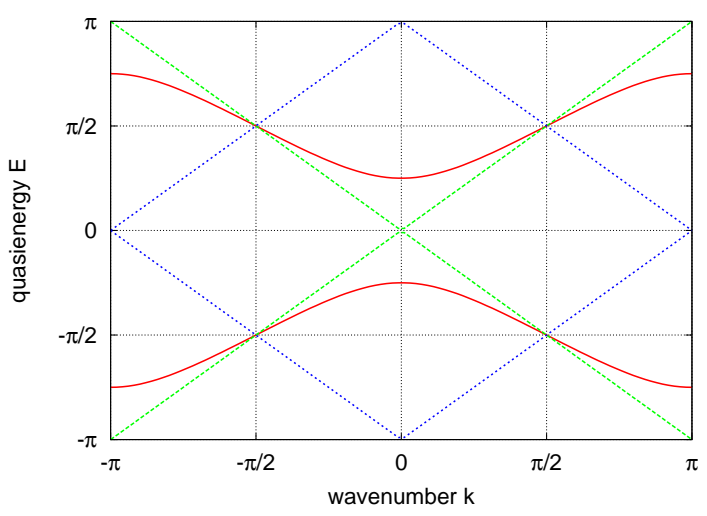

FIG. 2: Dispersion relations of the 1D quantum walk. Continuous line shows a typical gapped phase, with $\theta= \pm \pi / 4$. The two gapless dispersion relations are: $\theta=0$ (slashed) and $\theta= \pm \pi$ (dotted). In both cases the gaps at $E=0$ and $E= \pm \pi$ are closed.

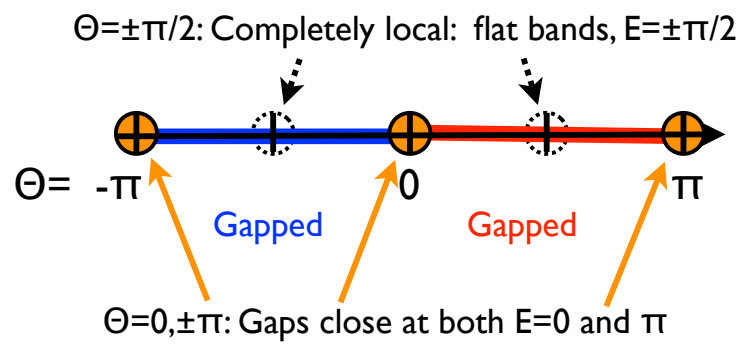

FIG. 3: Parameter space of the 1-dimensional simple quantum walk. The only parameter is the coin rotation angle $\theta$. The parameter space consists of two gapped domains, with gaps around both $E=0$ and $E=\pi$. These are separated by the gapless points, $\theta=0$, and $\theta=\pi$.

in Fig. 2. Note that for generic rotation angle $\theta$, the dispersion relation has gaps around $E=0$ and around $E=\pi$.

At the time reversal invariant momenta $k=0$ and $k=\pi$, the Floquet operator $U(k)$, as in Eq. (9), has a particularly simple form:

$$
\begin{aligned}
U(k=0) & =e^{-i \theta \sigma_{y}} \\
U(k=\pi) & =e^{-i\left(\pi+\theta \sigma_{y}\right)} .
\end{aligned}
$$

This shows directly that the dispersion relation has gaps at $k=0, E=0$ and at $k=\pi, E=\pi$ of magnitude $\theta$. Thus, the parameter space $\theta=\pi, \ldots, \pi$ falls apart to two disconnencted intervals where the system is gapped: $-\pi<\theta<0$ and $0<\theta<\pi$. This is illustrated in Fig. 3. This allows for the possibility that these regimes correspond to two distinct topological phases. 


\section{A. Edge states in the simple quantum walk}

Whether the simple quantum walks with $0<\theta<\pi$ and $-\pi<\theta<0$ constitute different topological phases can be checked by considering an inhomogeneous system. As an illustration, we show a simple choice, a quantum walk on $N=40$ sites, with $U=S R\left(\theta_{A}, \theta_{B}\right)$, where the rotation operator reads

$$
R\left(\theta_{A}, \theta_{B}\right)=\sum_{x \in A}|x\rangle\left\langle x\left|\otimes e^{-i \theta_{A} \sigma_{y}}+\sum_{x \notin A}\right| x\right\rangle\langle x| \otimes e^{-i \theta_{B} \sigma_{y}} .
$$

The domain with rotation angle $\theta_{A}$ is defined by

$$
x \in A \Leftrightarrow 10<x \leq 30 .
$$

We start the walker localized at $x=10$, with spin up. As shown in Fig. 4, when $\theta_{A}=\theta_{B}$, the walker spreads, with the maximum of the probability spreading with the maximum of the group velocity. If $\theta_{A}$ and $\theta_{B}$ are different, but in the same phase, there are diffraction effects at the boundaries $x=10$ and $x=30$. If $\theta_{B}$ and $\theta_{A}$ have different signs, a part of the walker is localized at the boundary at $x=10$.

Observed more closely, it is apparent that the walker trapped at the domain boundary in Fig. 44) performs a "zigzag" motion. This is a consequence of the fact that there are not one, but two localized states at the interface. These are SLS partners of each other, and thus 1) their energies differ by $E=\pi$ and 2) that their wavefunctions are related by multiplication by $\tau_{z}$, the sublattice symmetry operator defined in Eq. (13). This zigzag motion has already been seen in experiment 14 , and its origin in the existence of two bound states has also been inferred. We now clarify the fact that it is sublattice symmetry that ensures that bound states always come in pairs of $E=0$ and $E=\pi$.

The existence of a pair of topologically protected bound states can be inferred based on the "adiabatic continuation" argument, as, e.g., in Kitagawa's pedagogical paper $\frac{13}{}$. To obtain a more complete picture, we need to find the topological invariants associated with the gapped phases.

\section{B. Topological invariants $Q_{0}, Q_{\pi}$}

Topological invariants for periodically modulated quantum systems have been suggested by Jiang et al! $\stackrel{19}{ }$, via an elaborate construction. For a periodically driven chain with particle-hole symmetric effective Hamiltonian, they suggest a $\mathbb{Z}_{2} \times \mathbb{Z}_{2}$ topological invariant, $\left(Q_{0}, Q_{\pi}\right)$. To define the invariant, they introduce a set of time dependent Hamiltonians $H_{T}(t)$, with the parameter $0 \leq T \leq 1$ specifying the time period. $H_{T}(t)$ should be a smooth function of $T$, with

$$
\begin{aligned}
& H_{T=0}(t)=0 ; \\
& H_{T=1}(t)=H(t) .
\end{aligned}
$$
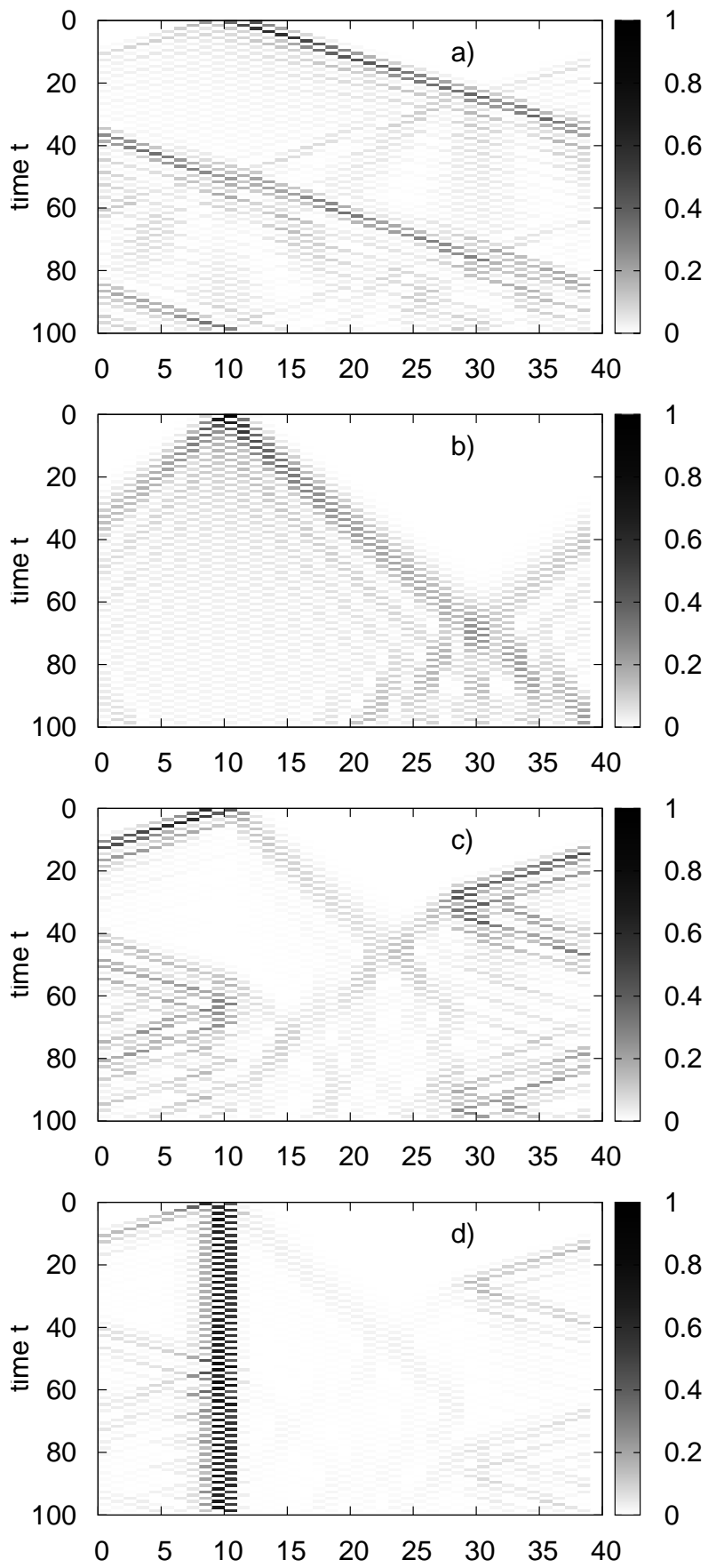

FIG. 4: Time dependence of the probability distribution (color coding corresponding to $\left|\Psi_{x, \uparrow}\right|^{2}+\left|\Psi_{x, \downarrow}\right|^{2}$ ) of a walker on a lattice consisting of $N=40$ sites with periodic boundary conditions. The coin operator is taken as $R(\theta(x))$, with $\theta(x)=\theta_{A}$ for $10<x<31$ and $\theta(x)=\theta_{B}$ otherwise. In each case we start the walker localized on the boundary betwen the domains of $\theta_{A}$ and $\theta_{B}$, on site $x=10$, with spin up. In cases a) and b), the walk is homogeneous: in a) $\theta_{A}=\theta_{B}=0.4 \pi$, and in b) $\theta_{A}=\theta_{B}=0.2 \pi$. In c), an inhomogenous system is considered, with a sharp boundary between two bulks with the same topology: $\theta_{A}=0.2 \pi$ and $\theta_{B}=0.4 \pi$. In d): $\theta_{A}=-0.2 \pi$ and $\theta_{B}=0.4 \pi$ : Here the two domains have different topology. Accordingly, a significant part ot the wavefunction of the walker gets trapped at the interface. 
To each $H_{T}(t)$ we can define the corresponding Floquet operator $U_{T}=\mathbb{T} e^{-i \int_{0}^{T} H_{T}(t) d t}$, and the corresponding effective Hamiltonian $H_{\mathrm{eff}, T}$. In the bulk, $H_{\mathrm{eff}, T}$ is translation invariant, and has a spectrum $E_{T}^{(n)}(k)$.

The next step to obtain the topological invariant $Q_{0}$, is to count the parity of the number of times the gap at $E=$ 0 closes during the path $T=0 \rightarrow 1$. Because of ParticleHole Symmetry, gaps at $\pm k$ close at the same time. It is therefore enough to count the number of solutions to $E_{T}^{(n)}(k=0)=m \pi$ with arbitrary even integer $m$, add to this the number of solutions to $E_{T}^{(n)}(k=\pi)=m \pi$ with arbitrary even integer $m$, divide the sum by 2 (gaps close from both directions), and take modulo 2 of the result. Repeating the same process for arbitrary odd integers $m$ gives us the invariant $Q_{\pi}$.

For the quantum walk, we can evaluate the topological invariants $Q_{\pi}$ and $Q_{0}$ without following the elaborate construction of Jiang et al. As shown in Appendix B as long as we are only interested in the differences between the topological invariants of two phases, say $A$ and $B$ - and this is all that matters for the physics - there is a quite straightforward method. 1) Select any point in parameter space representing a phase $A, 2$ ) connect it via a continuous path in parameter space to a representative point for phase $B$. 3) Count the parity of the number of times the gap around $E=0$ closes along this path, to obtain the invariant $Q_{0}$, and 4) similarly for the gap around $E= \pm \pi$ to obtain $Q_{\pi}$. This construction shows that the gapped phases $-\pi<\theta<0$ and $0<\theta<\pi$ differ in both invariants $Q_{0}$ and $Q_{\pi}$. This completes the bulkboundary correspondence picture for the edge states at the interfaces between these phases.

\section{QUANTUM WALK ON A FINITE LINE}

To have a discrete time quantum walk on a finite line, we need to terminate the $1 \mathrm{D}$ lattice. There are two ways to accomplish this: 1) changing the coin operators at the boundaries or 2) Cutting the bonds with reflection.

\section{A. Reflective Coin}

Totally reflective coins have already been considered in the literature. Obuse and Kawakam: ${ }^{15}$ mention that $\theta=-\pi / 2$ gives a reflective coin with edge states if the bulk has $\theta>0$ and $\theta=+\pi / 2$ should be taken for $\theta<0$. Kitagawa ${ }^{13}$ explains why this is so using an adiabatic continuation argument. For the sake of completeness we briefly summarize a different derivation here.

The totally reflective unitary coin operator reads

$$
R_{0}=\left(\begin{array}{cc}
0 & e^{i \phi} \\
e^{i \xi} & 0
\end{array}\right) .
$$

To keep Particle-Hole Symmetry represented by complex conjugation, we would like to choose $R_{0}$ to have only real elements. That leaves us 4 choices for $R_{0}: \pm \sigma_{x}$ and $\pm i \sigma_{y}$. A walker only sees the totally reflective coin from one side, and thus we can take $R= \pm i \sigma_{y}$ without loss of generality. This corresponds to choosing the reflective coin to have a rotation angle $\theta$ which is in the middle of one of the gapped phases. If this is the same gapped phase as that of the bulk, there are no bound states at the boundary. If it is not the same as that of the bulk, there are two bound states with energies 0 and $\pi$, that are sublattice partners of each other.

\section{B. Cutting a link}

Unitarity of the quantum walk is a strong constraint on how we can cut a link. When the walker attempts to jump over a link that is cut, it has to end up in a state which is unaccessible to it from any other state. The only states that are "not taken" are those to either sides of a cut link. Therefore, the only option to implement a totally cut link, is to introduce a spin flip instead of a jump. It is still possible to include a phase shift along with the spin flip. To retain PHS, this phase shift can only be chosen to be \pm 1 . In much the same way as with the reflective coin above, without loss of generality, we can fix a phase of -1 upon reflection from one of the sides. Cutting the link between sites $y$ and $y+1$ is implemented by altering the shift operator $S$ :

$$
S_{(y)}=\sum_{x \neq y} S_{x, x+1} \pm C_{y, y+1} .
$$

Here, the shift operators for the "link" and "cut link" between sites $x$ and $x+1$ are defined as

$$
\begin{aligned}
S_{x, x+1} & =|x, \downarrow\rangle\langle x+1, \downarrow|+| x+1, \uparrow\rangle\langle x, \uparrow| ; \\
C_{x, x+1} & =|x+1, \uparrow\rangle\langle x+1, \downarrow|-| x, \downarrow\rangle\langle x, \uparrow| .
\end{aligned}
$$

The \pm in Eq. 26) represents the choice of the reflection phases allowed by Particle-Hole symmetry.

\section{Partially cut links in the bulk}

In order to use bulk-boundary correspondence, we need to connect the "cut link" to the "uncut link" by way of a continuous parameter in the Floquet operator. The first idea here, the introduction of an additional "link rotation angle" $\phi$, works:

$$
S_{x, x+1}(\phi)=\cos (\phi) S_{x, x+1}+\sin (\phi) C_{x, x+1} .
$$

In the bulk, this is equivalent to the "split-step" walk of Kitagawa et $a^{[12}$, where the spin- $z$ dependent displacement is broken down to two successive steps:

$$
\begin{aligned}
& S_{\downarrow}=\sum_{x=1}^{N}(|x-1\rangle\langle x|\otimes| \downarrow\rangle\langle\downarrow|+| x\rangle\langle x|\otimes| \uparrow\rangle\langle\uparrow|) ; \\
& S_{\uparrow}=\sum_{x=1}^{N}(|x\rangle\langle x|\otimes| \downarrow\rangle\langle\downarrow|+| x+1\rangle\langle x|\otimes| \uparrow\rangle\langle\uparrow|) ;
\end{aligned}
$$




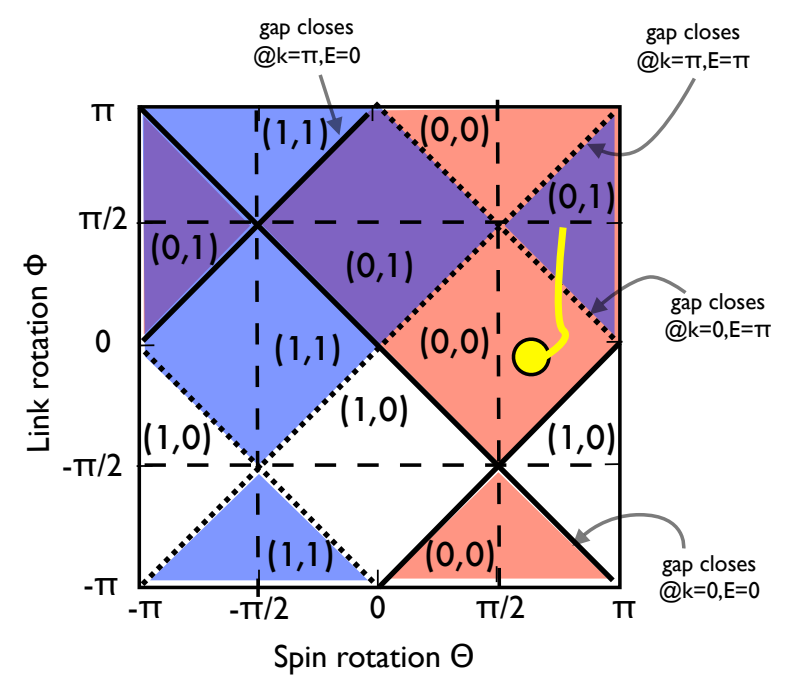

FIG. 5: Phase map of a 1D quantum walk with partially cut links. The various gapped domains (different shadings) have a $\mathbb{Z}_{2} \times \mathbb{Z}_{2}$ invariant associated with them, which is indicated as a pair of numbers $\left(Q_{0}, Q_{\pi}\right)$. Separating these domains are the lines where the gap at $E=0$ closes (continuous line), or the gap at $E=\pi$ closes (dotted line). closes. The vertical (horizontal) slashed lines denote the parameters corresponding to reflective coins (cut links). To find the number of edge states at an edge with cut links, a the point corresponding to the quantum walk (circle) is connected to the horizontal slashed line representing the boundary conditions. In the example shown, there is a single bound state with $E=\pi$.

$$
U_{2}(\theta, \phi)=S(\phi) R(\theta)=S_{\uparrow} e^{-i \phi \sigma_{y}} S_{\downarrow} e^{-i \theta \sigma_{y}} .
$$

As shown in $\frac{12}{2}, S(\phi=0)=S_{\uparrow} S_{\downarrow}=S$.

With a partially cut link, the sublattice symmetry of the Floquet operator $U$ is broken. A walker that is reflected off an edge has the same $x$ at the end of the timestep as at the beginning (and possibly even the same spin), therefore the graph of $U$ cannot be bipartite. Therefore, the gaps at $E=0$ and $E= \pm \pi$ now can open and close independently (and the energy $E= \pm \pi / 2$ is no longer protected by symmetries).

\section{Topological phases of the split-step walk}

The split-step quantum walk, Eq. (32), has two parameters, the "coin angle" $\theta$ and the "bond angle" $\phi$. The parameter space is therefore now a torus. The Floquet timestep operator $U_{2}$ reads

$$
U_{2}(k)=e^{-i \sigma_{z} k / 2} e^{-i \sigma_{y} \phi} e^{-i \sigma_{z} k / 2} e^{-i \sigma_{y} \theta} .
$$

The dispersion relation is straightforwardly derived ${ }^{12}$,

$$
\cos E(k)=\cos (k) \cos (\theta) \cos (\phi)-\sin (\theta) \sin (\phi)
$$

As can be seen from this dispersion relation, the splitstep quantum walk for generic $\phi$ and $\theta$ has gaps around
$E=0$ and $E= \pm \pi$. The Floquet operator takes on a very simple form at the time-reversal invariant momenta $k=0, \pi$ :

$$
\begin{gathered}
U_{2}(k=0)=e^{-i H(k=0)}=e^{-i \sigma_{y}(\theta+\phi)} \\
U_{2}(k=\pi)=e^{-i H(k=\pi)}=-e^{-i \sigma_{y}(\theta-\phi)} ;
\end{gathered}
$$

Therefore the gap around $E=0$ closes at $k=0, \phi=-\theta$, and $k=\pi, \phi= \pm \pi+\theta$, and the gap around $E= \pm \pi$ closes at $k=\pi, \phi=\theta$, and $k=0, \phi= \pm \pi-\theta$.

The parameter space $(\theta, \phi)$ is divided into 4 different gapped topological phases, with topological invariants $Q_{0}$ and $Q_{\pi}$, as shown in Fig. 5. Selecting $\theta=\pi / 2, \phi=0$ as a reference point, we define the values of the invariants for the domain around this point as $(0,0)$. For any point in parameter space, we 1) pick a continuous path in parameter space connecting it with the reference point, 2 ) count the parity of the number of times gap around $E=0$ $(E=\pi)$ closes along the path. The parities give the values of the invariant $Q_{0}\left(Q_{\pi}\right)$. Because of Particle-Hole Symmetry, it is enough to count the gap closings at the time-reversal invariant momenta $k=0$ and $k= \pm \pi$.

Setting $\phi=0$ corresponds to the original "simple" quantum walk. Setting $\phi= \pm \pi / 2$ corresponds to two different ways in which the bonds can be cut in a unitary and particle-hole-symmetric way. As illustrated in Fig 5 , using the bulk-boundary correspondence, we find that for a generic quantum walk with $\phi=0$, each edge defined by cutting a link in a particle-hole-symmetric way hosts a single topologically protected edge state. Whether the energy of that state is $E=0$ or $E=\pi$ depends on the bulk quantum walk and on the way in which the link is cut (on the reflection phase). In the example of 5 , we find that the energy of the bound state is $E=\pi$. Note that this is independent of the path itself, and also of its endpoint on the line representing the cut links - except if this endpoint is at $\theta= \pm \pi / 2$, in which case the details of the edge need to be specified to show whether the reflection is off of the reflective coin or from the cut link. This is illustrated in Fig. 6.

\section{E. Edge states between two bulks with the same Floquet operator}

The topological invariant for a quantum walk cannot be inferred from its effective Hamiltonian alone. Evidence for this has already been noted by Kitagawa ${ }^{13}$, who describes pairs of bound states between topological phases with the same "winding number". The most striking illustration of this statement, however, is a pair of edge states between two bulks with the same Floquet operator.

Consider an inhomogeneous quantum walk with periodic boundary conditions, consisting of two bulks, separated by a sharp boundary. The dynamics is given by the split-step protocol, and the bulks differ in both pa- 


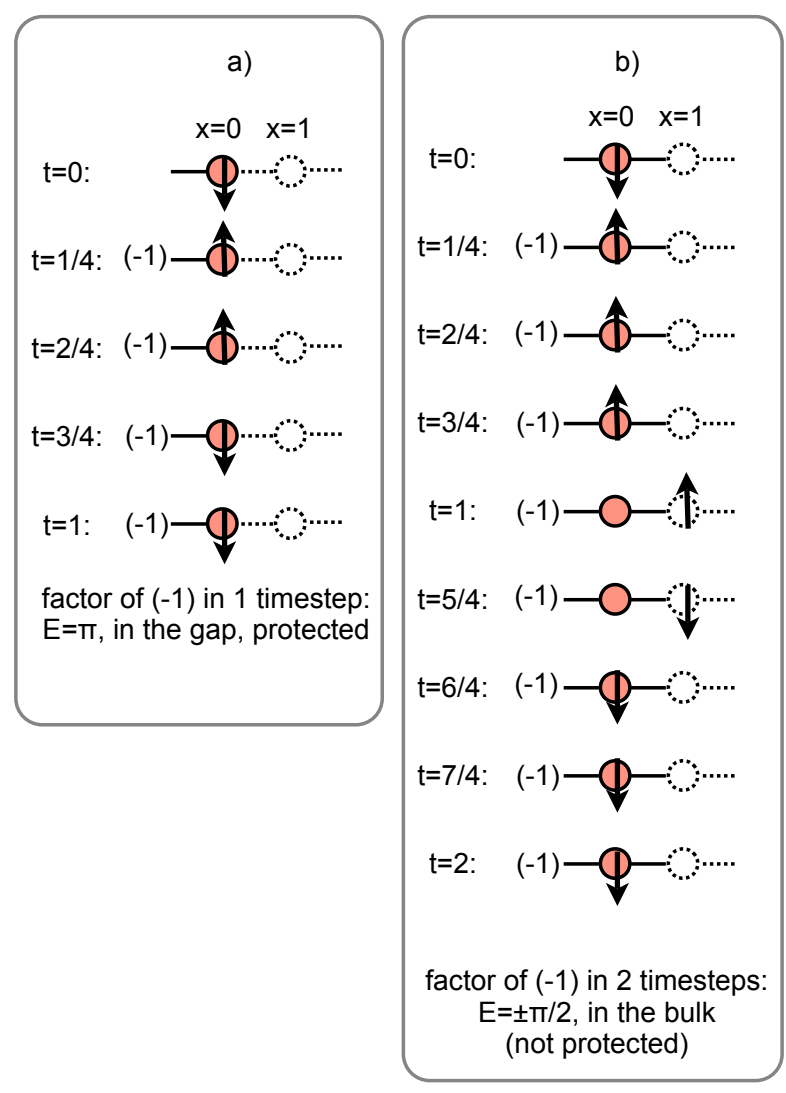

FIG. 6: Timesteps for a walker started from an edge. Contionuous (dotted) circles and lines correspond to the sites and links of the bulk (boundary). The timesteps are broken down to 4 successive operations, as in Eq. (33), each occurring in $1 / 4$ time. Continuous (dotted) circles and lines correspond to the sites and links of the bulk (boundary). For simplicity, the bulk is taken with $\theta=\pi / 2$, and $\phi=0$ : a simple quantum walk. The boundary has $\theta=\pi / 2$, and cut links: $\phi=\pi / 2$. If the reflection is on a cut link (a), there is a protected midgap edge state with energy $\pi$. If the reflection happens on a reflective coin (b), during two timesteps, the walker acquires a phase of (-1). Superpositions of the states at $t=0$ and $t=1$ with a relative phase of $i(-i)$ are therefore stationary states with energy $-\pi / 2(\pi / 2)$, not protected by particle-hole symmetry.

rameters $\theta$ and $\phi$ :

$$
U_{2}=S_{\uparrow} R\left(\phi_{A}, \phi_{B}\right) S_{\downarrow} R\left(\theta_{A}, \theta_{B}\right),
$$

with the inhomogeneous rotation operator $R\left(\theta_{A}, \theta_{B}\right)$ defined as in Eq. 21. Taking $\phi_{B}=\phi_{A}+\pi$ and $\theta_{B}=\theta_{A}+\pi$, the translationally invariant bulk time evolution operators of the two domains read

$$
\begin{aligned}
U_{A} & =S_{\uparrow} e^{-i \phi_{A} \sigma_{y}} S_{\downarrow} e^{-i \theta_{A} \sigma_{y}} \\
U_{B} & =S_{\uparrow} e^{-i\left(\phi_{A}+\pi\right) \sigma_{y}} S_{\downarrow} e^{-i\left(\theta_{A}+\pi\right) \sigma_{y}} .
\end{aligned}
$$

Note that since $e^{-i \pi \sigma_{y}}=-1$, we have

$$
U_{A}=U_{B}
$$

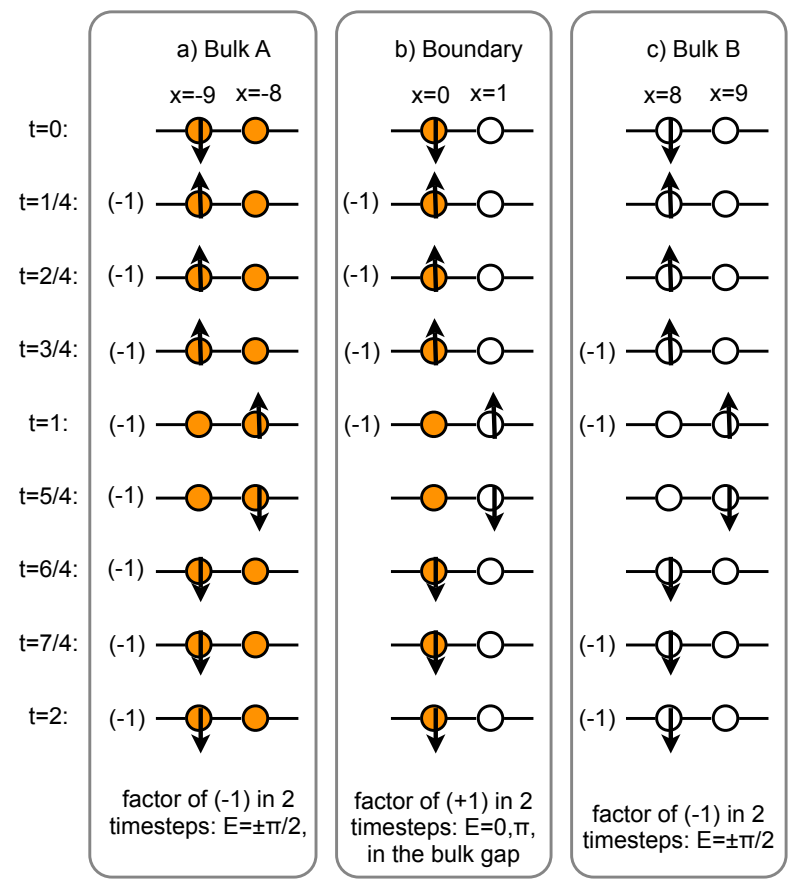

FIG. 7: Two successive timesteps of a quantum walk, with a walker started in bulk A (a), at a sharp boundary (b), or in bulk B (c). Each timestep is broken down to its 4 stages, given by the 4 factors in $U_{2}(k)=S_{\uparrow} R(0, \pi) S_{\downarrow} R(\pi / 2,-\pi / 2)$, with $R\left(\theta_{A}, \theta_{B}\right)$ as defined in Eq. 21], with $x \in A \leftrightarrow x<$ 1. In each case, the walker returns to its initial site after 2 timesteps. In the bulk, during the 2 timesteps a phase factor of $(-1)$ is acquired by the walker, showing that stationary states (superpositions of the states at $t=0$ and $t=1$ with relative phase $\pm i$ ) have quasienergy $\mp \pi$. At the boundary, this factor is $(+1)$, therefore even and odd superpositions of the states at $t=0$ and $t=1$ are stationary states with energy $0, \pi$. These are at the topologically protected midgap states.

As can be seen from the phase map, Fig 5 , the simplest path in the parameter space connecting two such points intersects gap closings at $E=0$ and at $E=\pi$ once. Thus, there are 2 edge states between these two bulks, with energies 0 and $\pi$.

Perhaps the simplest concrete example is a boundary between $\phi=0, \theta=\pi / 2$, and $\phi=\pi, \theta=-\pi / 2$. We illustrate this in Fig. 7 .

\section{CONCLUSIONS}

In this paper we revisited the topological phases of the 1-dimensional quantum walk. To begin, we identified the symmetries of the corresponding effective Hamiltonian. In contrast with the literature, we find that the Hamiltonian belongs to class D, i.e., it has a ParticleHole Symmetry that squares to 1 , and no other symmetries. We argue that the property of the homogeneous quantum walk identified as Chiral Symmetry should not 
rightfully be regarded as a symmetry, since the operator representing it varies from phase to phase. We also find that there is an additional symmetry of the timestep operator, which could be called "sublattice symmetry", however, it does not have any special effect on the topological properties of the system.

To identify the topological phases of 1-dimensional discrete-time quantum walks, however, the bulk effective Hamiltonian (or indeed, the bulk Floquet operator) is not enough. We have found that a more complete specification of the experimental realization is needed, e.g., the sequences of rotation-translation. We have evaluated the topological invariant of Jiang et al ${ }^{[19}$ for such specifications corresponding to the simple discrete-time quantum walk, and for the "split-step" walk introduced by Kitagawa et al.12. For the simple walk we find two different phases, whose boundary hosts a pair of topologically protected edge states. For the split-step walk, we find all 4 different topological phases corresponding to the $\mathbb{Z}_{2} \times \mathbb{Z}_{2}$. We provide a blatant proof of the fact that the bulk Floquet operator does not contain all the information about the topological phase: A pair of topologically protected edge states between two bulks which differ in their experimental description, but have the same Floquet operator.

The use of periodically modulated external fields to alter the topological properties of Hamiltonians has been considered by several authors 2122 . In all cases, however, these works employ the same topological invariants as for time-independent systems. It would be interesting to explore what the complete topological invariant in these cases is, and under what conditions does it give rise to edge states that are unique to periodically driven systems. Kitagawa et al ${ }^{17}$ have already shown that for a periodically modulated hexagonal lattice, edge states can arise between bulk phases with the same Chern number. However, even for this specific system, the bulk topological invariant has not yet been defined.

This work was supported by the Hungarian Academy of Sciences (Lendület Program, LP2011-016). We acknowledge useful discussions with Anton Akhmerov, Tamás Kiss, and Zoltán Kurucz.

\section{Appendix A: Sublattice symmetry and the doubling of states}

Any stationary state $|\Psi\rangle$ of a quantum walk with sublattice symmetry must have support on both sublattices $A$ and $B$. Using the obvious notation for the projection of a state on a sublattice, $\left|\Psi_{A}\right\rangle \equiv \sum_{j \in A}|j\rangle\langle j|| \Psi\rangle$, and similarly, $\left|\Psi_{B}\right\rangle \equiv \sum_{j \in B}|j\rangle\langle j \| \Psi\rangle$, we have

$$
|\Psi\rangle=\left|\Psi_{A}\right\rangle+\left|\Psi_{B}\right\rangle
$$

For stationary states, $U|\Psi\rangle=e^{-i E}|\Psi\rangle$, which gives us $U\left|\Psi_{A, B}\right\rangle=e^{-i E}\left|\Psi_{B, A}\right\rangle$. Therefore, both $\left|\Psi_{A}\right\rangle$ and $\left|\Psi_{B}\right\rangle$ are eigenstates of the step-doubled walk,

$$
U^{2}\left|\Psi_{A, B}\right\rangle=e^{-2 i E}\left|\Psi_{A, B}\right\rangle .
$$

Doubling the timestep gives a walk on only one sublattice, since $U^{2} \tau_{z}=\tau_{z} \tau_{z} U \tau_{z} \tau_{z} U \tau_{z}=\tau_{z} U^{2}$, and projection to sublattice $A, B$ is given by $1 / 2\left(1 \pm \tau_{z}\right)$. Therefore, we can double the timestep and restrict to sublattice $A$. For any eigenstate of $U^{2}$ with energy $E_{2}$, we have :

$$
\begin{aligned}
U\left|\Psi_{A}\right\rangle & =\left|\Psi_{B}\right\rangle ; \\
U\left|\Psi_{B}\right\rangle & =e^{-i E_{2}}\left|\Psi_{A}\right\rangle ;
\end{aligned}
$$

Introducing $E=E_{2} / 2$, we can reconstruct the two eigenstates of $U$ with energies $E_{2}$ and $E_{2}+\pi$, linked by sublattice symmetry:

$$
U\left(\left|\Psi_{A}\right\rangle \pm e^{-i E}\left|\Psi_{B}\right\rangle\right)= \pm e^{-i E}\left(|\Psi\rangle_{A} \pm e^{-i E}\left|\Psi_{B}\right\rangle\right) .
$$

Therefore, any energy eigenstate of $U^{2}$, projected onto one of the sublattices, gives us two energy eigenstates of $U$, related to each other by the sublattice symmetry. This means that we can double the timestep without losing any energy eigenstates.

\section{Appendix B: The $\mathbb{Z}_{2} \times \mathbb{Z}_{2}$ invariant in parameter space}

To infer the number of topologically protected edge states at an edge between two bulks $A$ and $B$, i.e., to apply the bulk-boundary correspondence, we do not need to know the values of the topological invariants $\left(Q_{0}, Q_{\pi}\right)$ in these bulks. It is enough to know the amounts by which the values of these invariants change between the two bulks. Therefore it is not necessary to find the complete set $H_{T}(t)$, corresponding to a continuous path in parameter space to "doing nothing".

We assume two things. First, that a set of experimental Hamiltonians exists for bulk $A$ that connects it to "doing nothing": $H_{T}^{A}(t)$, with $H_{T=0}^{A}=1$ and $H_{T=1}^{A}(t)=H_{\exp }^{A}$. Second, that for the continuous path in the space of parameters of the quantum walk, $\theta(x)$, with $\theta(x=0)=\theta_{A}$ and $\theta(x=1)=\theta_{B}$, the experimental Hamiltonians $H^{\theta}(x)_{\exp }$ along the path are also continuous functions of $x$.

We construct the path $H_{\exp , T}^{B}$ in the following way:

$$
\begin{aligned}
& H_{\exp , T}^{B}(t)=H_{\exp , 2 T}^{A}(2 t) \quad \text { if } \quad T<1 / 2 \\
& H_{\exp , T}^{A}(t)=H_{\exp }^{\theta(2 T-1)}(t / T) \quad \text { if } \quad T \geq 1 / 2 .
\end{aligned}
$$

For this construction, the difference in the invariant $Q_{0}$ between the bulks $B$ and $A$ can be obtained by just counting the number of times the gap around $E=0$ closes along the path $\theta(x)$. The analogous recipe holds for the invariant $Q_{\pi}$, with the gap around $E= \pm \pi$. 
1 Y. Aharonov, L. Davidovich, and N. Zagury, Phys. Rev. A 48, 1687 (1993), URL http://link.aps.org/doi/10. 1103/PhysRevA.48.1687.

${ }^{2}$ A. M. Childs, Phys. Rev. Lett. 102, 180501 (2009), URL http://link.aps.org/doi/10.1103/PhysRevLett. 102.180501

3 A. M. Childs, D. Gosset, and Z. Webb, arxiv p. arXiv:1205.3782v1 (2012).

${ }^{4} \mathrm{~V}$. Kendon, Mathematical Structures in Computer Science 17, 1169 (2007).

${ }^{5}$ F. Zähringer, G. Kirchmair, R. Gerritsma, E. Solano, R. Blatt, and C. F. Roos, Phys. Rev. Lett. 104, 100503 (2010), URL http://link.aps.org/doi/10.1103/ PhysRevLett.104.100503.

${ }^{\circ}$ H. Schmitz, R. Matjeschk, C. Schneider, J. Glueckert, M. Enderlein, T. Huber, and T. Schaetz, Phys. Rev. Lett. 103, 090504 (2009), URL http://link .aps .org/doi/10. 1103/PhysRevLett.103.090504

M. Karski, L. Förster, J.-M. Choi, A. Steffen, W. Alt, D. Meschede, and A. Widera, Science 325, 174 (2009), http://www.sciencemag.org/content/325/5937/174.full.pdf, URL http://www.sciencemag.org/content/325/5937/ 174.abstract

8 A. Schreiber, K. N. Cassemiro, V. Potoček, A. Gábris, P. J. Mosley, E. Andersson, I. Jex, and C. Silberhorn, Phys. Rev. Lett. 104, 050502 (2010), URL http://link.aps. org/doi/10.1103/PhysRevLett.104.050502

${ }^{9}$ M. A. Broome, A. Fedrizzi, B. P. Lanyon, I. Kassal, A. Aspuru-Guzik, and A. G. White, Phys. Rev. Lett. 104, 153602 (2010), URL http://link.aps .org/doi/10.1103/ PhysRevLett.104.153602.

I0 X.-L. Qi and S.-C. Zhang, Rev. Mod. Phys. 83, 1057 (2011), URL http://link.aps.org/doi/10.1103/ RevModPhys.83.1057

${ }^{11}$ M. Z. Hasan and C. L. Kane, Rev. Mod. Phys. 82,
3045 (2010), URL http://link.aps.org/doi/10.1103/ RevModPhys.82.3045

${ }^{12}$ T. Kitagawa, M. S. Rudner, E. Berg, and E. Demler, Phys. Rev. A 82, 033429 (2010), URL http://link.aps.org/ doi/10.1103/PhysRevA.82.033429.

${ }^{13}$ T. Kitagawa, arxiv p. arXiv:1112.1882v1 (2011).

14 T. Kitagawa, M. A. Broome, A. Fedrizzi, M. S. Rudner, E. Berg, I. Kassal, A. Aspuru-Guzik, E. Demler, and A. G. White, arxiv p. arXiv:1105.5334v1 (2011).

15 H. Obuse and N. Kawakami, Phys. Rev. B 84, 195139 (2011), URL http://link.aps.org/doi/10.1103/ PhysRevB.84.195139.

16 P. W. Brouwer, A. Furusaki, I. A. Gruzberg, and C. Mudry, Phys. Rev. Lett. 85, 1064 (2000), URL http://link.aps . org/doi/10.1103/PhysRevLett.85.1064

Ir T. Kitagawa, E. Berg, M. Rudner, and E. Demler, Phys. Rev. B 82, 235114 (2010), URL http://link.aps.org/ doi/10.1103/PhysRevB.82.235114.

18 T. Oka, N. Konno, R. Arita, and H. Aoki, Phys. Rev. Lett. 94, 100602 (2005), URL http://link.aps.org/doi/10. 1103/PhysRevLett.94.100602

${ }^{19}$ L. Jiang, T. Kitagawa, J. Alicea, A. R. Akhmerov, D. Pekker, G. Refael, J. I. Cirac, E. Demler, M. D. Lukin, and P. Zoller, Phys. Rev. Lett. 106, 220402 (2011), URL http://link.aps.org/doi/10.1103/PhysRevLett. 106.220402

20 Y. Shikano and H. Katsura, Phys. Rev. E 82, 031122 (2010), URL http://link .aps .org/doi/10.1103/ PhysRevE. 82.031122.

${ }^{21}$ L. N. H., G. Refael, and V. Galitski, Nature Physics 7, 490495 (2011).

22 B. Dóra, J. Cayssol, F. Simon, and R. Moessner, Phys. Rev. Lett. 108, 056602 (2012), URL http://link.aps. org/doi/10.1103/PhysRevLett.108.056602. 